\title{
Gut microbiota differences between paired intestinal wall and digesta samples in three small species of fish
}

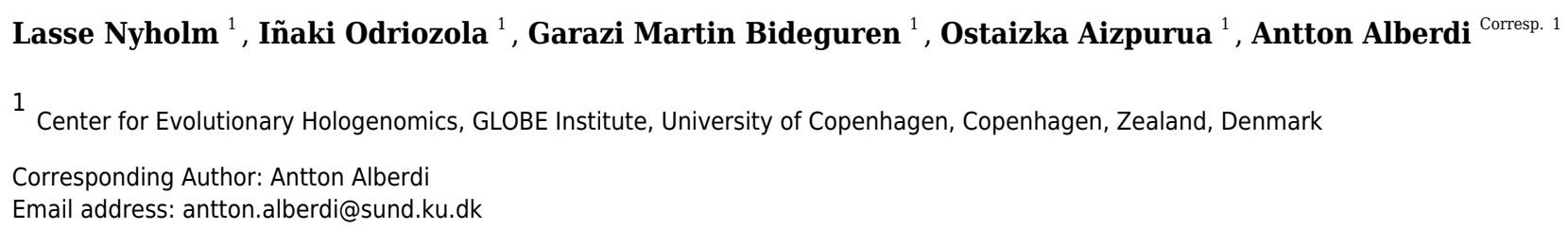

The microbial gut communities of fish are receiving increased attention for their relevance, among others, in a growing aquaculture industry. The members of these communities are often split into resident (long-term colonisers specialised to grow in and adhere to the gut) and transient (short-term colonisers originated from food items and the surrounding water) microorganisms. Separating these two communities in small fish are impeded by the small size and fragility of the gastrointestinal tract. With the aim of testing whether it is possible to recover two distinct communities in small species of fish using a simple sampling technique, we used $16 \mathrm{~S}$ amplicon sequencing of paired intestinal wall and digesta samples from three small Cyprinodontiformes fish. We examined the diversity and compositional variation of the two recovered communities, and we used joint species distribution modelling to identify microbes that are most likely to be a part of the resident community. For all three species we found that the diversity of intestinal wall samples was significantly lower compared to digesta samples and that the community composition between sample types was significantly different. Across the three species we found eight unique families of bacteria to be significantly enriched in samples from the intestinal wall, encompassing most of the 89 ASVs enriched in intestinal wall samples. We conclude that it is possible to characterise two different microbial communities and identify potentially resident microbes through separately analysing samples from the intestinal wall and digesta from small species of fish. We encourage researchers to be aware that different sampling procedures for gut microbiome characterization will capture different parts of the microbiome and that this should be taken into consideration when reporting results from such studies on small species of fish. 
1 Gut microbiota differences between paired

2 intestinal wall and digesta samples in three

3 small species of fish

4 Lasse Nyholm $^{1}$, Iñaki Odriozola ${ }^{1}$, Garazi Martin ${ }^{1}$, Ostaizka Aizpurua ${ }^{1}$ \& Antton Alberdi ${ }^{1, *}$

${ }^{1}$ Center for Evolutionary Hologenomics, GLOBE Institute, University of Copenhagen, Copenhagen, DK-1353, Denmark

${ }^{*}$ Corresponding author 


\section{Gut microbiota differences between paired}

6 intestinal wall and digesta samples in three

\section{7 small species of fish}

8 Abstract

9 The microbial gut communities of fish are receiving increased attention for their relevance, among others, in a growing aquaculture industry. The members of these communities are often split into resident (long-term colonisers specialised to grow in and adhere to the gut) and transient (shortterm colonisers originated from food items and the surrounding water) microorganisms. Separating these two communities in small fish are impeded by the small size and fragility of the gastrointestinal tract. With the aim of testing whether it is possible to recover two distinct communities in small species of fish using a simple sampling technique, we used 16S amplicon sequencing of paired intestinal wall and digesta samples from three small Cyprinodontiformes fish. We examined the diversity and compositional variation of the two recovered communities, and we used joint species distribution modelling to identify microbes that are most likely to be a part of the resident community. For all three species we found that the diversity of intestinal wall samples was significantly lower compared to digesta samples and that the community composition between sample types was significantly different. Across the three species we found seven unique families of bacteria to be significantly enriched in samples from the intestinal wall, encompassing most of the 89 ASVs enriched in intestinal wall samples. We conclude that it is possible to characterise two different microbial communities and identify potentially resident microbes through separately analysing samples from the intestinal wall and digesta from small species of fish. We encourage researchers to be aware that different sampling procedures for gut microbiome characterization will capture different parts of the microbiome and that this should be taken into consideration when reporting results from such studies on small species of fish. 


\section{Introduction}

30 Microbes found in the gastrointestinal (GI) tract of vertebrates can play an important role in

31 shaping their host's fitness (Barko et al., 2018; Compant et al., 2019; Legrand et al., 2020). Hence,

32 the study of these microbes and how they interact with their respective hosts are of high priority

33 for a better understanding of basic ecology questions as well as its implication in many applied

34 fields of research (Nyholm et al., 2020). However, some microbes are likely to influence their host

35 more than others based on how long they are found in the gut and how intimately they are 36 connected with the mucosa and mucus layer of their host (Kim, Brunt \& Austin, 2007; Ringø et

37 al., 2016; Gajardo et al., 2016). As a consequence, microorganisms found in the intestinal tract of

38 fish are often classified as transient (allochthonous) or resident (autochthonous) (Ringø et al.,

39 2003; Kim, Brunt \& Austin, 2007). Transient microbes are considered as "short-term colonisers"

40 passing through the gastrointestinal tract and are to a high degree influenced by environmental

41 factors (Legrand et al., 2020). In contrast, resident microbes are considered to be more or less

42 constantly present on the mucus surface of the intestine or in close proximity to epithelial cells

43 (Ringø et al., 2016) and believed to be affected by host genotype (Llewellyn et al., 2014; Bolnick

44 et al., 2014; Legrand et al., 2020) and the physical and chemical environment in and on the mucus

45 layer (Nayak, 2010). Previous studies addressing these two communities in teleost fish have found

46 that both communities are dominated by Proteobacteria, yet the resident community is less diverse

47 than the transient community (Kim, Brunt \& Austin, 2007; Wu et al., 2010; Gajardo et al., 2016;

48 Riiser et al., 2018). As a consequence, transient and resident microbial communities are likely to

49 vary, not only in their diversity, composition and abundance profiles, but also by the magnitude of

50 which they are influenced by their host and vice versa (Kim, Brunt \& Austin, 2007; Gajardo et al., 51 2016).

Setting a threshold for when a microbe is considered a transient visitor or a resident part of the host-associated microbiome is not straightforward, but specific sampling protocols can be employed for a better approximation to one or the other. For larger fish (e.g. Salmonids) it is common practice to collect the intestinal digesta to characterise the transient microbial community and then wash the intestine wall with saline buffer and then collect mucosal scrapes or swaps to characterise the resident microbial community (Kim, Brunt \& Austin, 2007; Feng et al., 2010; 
59 Gajardo et al., 2016). This is more difficult when handling smaller fish (e.g. zebrafish) as it is

60 rarely possible to open the intestine longitudinally and wash the intestinal lining with saline buffer

61 due to the size and fragility of the intestinal tissue. As a consequence, researchers working with

62 small species of fish or early life stages of larger fish will often sample the whole intestine

63 including digesta (Burns et al., 2017; Breen et al., 2019; Almeida, Domingues \& Henriques, 2021),

64 which hampers the distinguishing between transient and resident microbial communities. One

65 solution to reduce the impact of transient microbes when characterising the resident microbiome

66 of small captive fish is to collect the samples after a period without access to food (Lan \& Love,

67 2012; Sullam et al., 2012). However, this is not feasible when working on wild fish, as the chance

68 of distorting the "wild" microbiome is likely to increase with time an animal spends in captivity

69 (Restivo et al., 2021a) and time without access to food (Xia et al., 2014; Kohl et al., 2014; Mekuchi

70 et al., 2018; Li et al., 2019). An alternative method is to squeeze the digesta out of the intestine

71 and store the empty intestine (resident community) and digesta (transient community) separately. 72

73 To investigate the ability of the latter described sampling procedure to identify the transient and resident microbial communities of small fish species or early life stages of larger fish, we collected paired samples of the intestinal wall (a proxy for mucus-mucosa-resident community) and digesta from three small cyprinodontiform fish species from different locations. We used 16S amplicon sequencing to examine if this sampling protocol is able to identify whether 1) samples from the intestinal wall and digesta differ their diversity of microbes, 2) if the community structure is different between the intestinal wall and digesta and 3) if it is possible, even with spillover between sample types, to identify taxa that are likely to be a part of the resident microbiota.

\section{Materials and methods}

\section{Sample collection and storage}

83 Spanish toothcarps (Aphanius iberus, AI; n=27), Eastern mosquitofish (Gambusia holbrooki, GH; $84 \mathrm{n}=26$ ) and Valencian toothcarps (Valencia hispanica, VH; $\mathrm{n}=23$ ) were caught using baited minnow traps at 11 wetland locations in Valencia, Southeastern Spain, in August 2017 and September 2018

86 (Table S1) with permission from the Government of Valencia. These three species of fish were 
87 chosen as part of a larger project investigating the influence of the microbiome in species 88 conservation, and because they serve as good representatives of the challenges faced by researchers when sampling the gut microbiome of small wild fish species. To minimise contamination risk during dissection, fish were taken to a research facility while keeping them in

91 large (40L) oxygenated tanks separated by species and location with water from their natural 92 habitat for a maximum of four hours.

94 Right before dissection, fish were euthanized by a quick blow to the head in accordance with the Spanish law on animal research ethics (RD 53/2013) and the European Directive on the protection of animals used for scientific purposes (2010/63/EU). The entire intestine of each fish was extracted using scalpels and forceps in a sterilised environment and two types of samples were obtained (Fig. 1). Digesta samples (D, Fig. 1) were collected by squeezing the intestine using forceps and stored in absolute ethanol in individual tubes. As very little digesta was present in the samples, we used all available digesta from each fish as input for the extractions. The entire emptied intestines were used as a proxy for the intestinal wall microbial community (W, Fig. 1) and were cut into smaller pieces using sterile scalpels over sterile weighing boats and stored under identical conditions. Animals from which it was not possible to squeeze out any digesta of the dissected intestine were excluded from this study and are not a part of the total reported sample size. Samples were stored at $-20^{\circ} \mathrm{C}$ until DNA extraction.

\section{DNA extraction}

107 DNA was extracted using the tube-based Powersoil ${ }^{\oplus}$ DNA Isolation Kit (MoBio, CA, USA) 108 following the manufacturer's protocol with minor modifications (Table S2). DNA was eluted in

$10950 \mu \mathrm{EB}$ buffer and stored at $-20^{\circ} \mathrm{C}$. Extraction blanks were included in each extraction round to 110 screen for potential contamination. All extractions were done in a dedicated pre-PCR laboratory.

\section{PCR, library build and sequencing}

112 The generation of sequencing data and its subsequent bioinformatic processing were done similar 113 to what has previously been described in Aizpurua et al. (2021). Specifically, PCR amplification 114 of the V3-V4 region of the bacterial 16S rRNA gene was done using a broadly validated primer 
115 set (341F-805R) (Muyzer, de Waal \& Uitierlinden, 1993; Caporaso et al., 2011). Addition of 24

116 different tags to the 5 '-end of both primers enabled the differentiation of pooled samples after

117 sequencing (Binladen et al., 2007). For each sample three technical replicates were PCR amplified

118 to minimise the effect of PCR stochasticity (Alberdi et al., 2017). PCR amplifications were done

119 using an Applied Biosystems 2720 Thermal Cycler with a total reaction volume of $25 \mu 1$ consisting

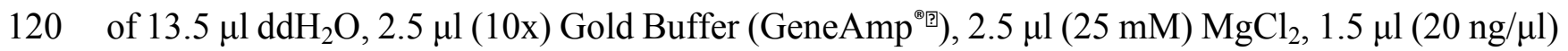

121 BSA, 0.5 dNTPs $(10 \mathrm{mM}), 0.5 \mu \mathrm{l}(5 \mathrm{U} / \mu \mathrm{l})$ DNA polymerase (AmpliTaq Gold $\left.{ }^{\oplus}\right), 2 \mu \mathrm{l}(10 \mathrm{mM})$

122 primer mix (forward and reverse) and $2 \mu \mathrm{LNA}$ extract. PCR settings were $95^{\circ} \mathrm{C}$ for 10 minutes,

12330 cycles of $95^{\circ} \mathrm{C}$ for 15 seconds, $53^{\circ} \mathrm{C}$ for 20 seconds and $72^{\circ} \mathrm{C}$ for 40 seconds and $72^{\circ} \mathrm{C}$ for 10

124 minutes. PCR products were visualised on $2 \%$ agarose gel by loading a mix of $4 \mu$ PCR product

125 with $2 \mu$ l loading buffer and given a score (0-3) based on band strength. Based on the band strength

126 scores $(0=10 \mu \mathrm{l}, 1=10 \mu \mathrm{l}, 2=8 \mu \mathrm{l}$ and $3=10 \mu \mathrm{l})$ the PCR products were pooled together in pools

127 consisting of 24 unique tags allowing the tracking of each sample back to individual fish (Binladen

128 et al., 2007). Amplicon pools were subsequently purified using SPRI beads (DeAngelis, Wang \&

129 Hawkins, 1995; Rohland \& Reich, 2012) in a 1:1 beads:DNA ratio to remove non-target DNA and

130 primer dimers. Amplicon pools were quantified on a Qubit (Thermo Fisher Scientific ${ }^{0}$ ) and

131 diluted in $30 \mu \mathrm{l}$ water to a total amount of $500 \mathrm{ng}$ of DNA. Libraries were constructed using the

132 Blunt-End Single-Tube Tagsteady protocol described by (Carøe \& Bohmann, 2020) and quantified

133 through qPCR by mixing $2 \mu 1$ 1:10,000 dilution of each library with $8 \mu$ Quant Mastermix with

134 added primers (NEB) and running it for 1 cycle of $95^{\circ} \mathrm{C}$ for 1 minutes, 35 cycles of $95^{\circ} \mathrm{C}$ for 15

135 seconds and $63^{\circ} \mathrm{C}$ for 45 seconds on a Stratagene Mx3006P (Agilent Technologies ${ }^{\complement \varpi}$ ). Different

136 volumes of each library were pooled together to ensure equal molarity and sequenced on an

137 Illumina MiSeq platform aiming at 9,000-62,000 reads per PCR replicate.

\section{Bioinformatics}

139 Paired-end reads were demultiplexed based on library indices and trimmed using AdapterRemoval

$140 \quad 2.2 .2$ (Schubert, Lindgreen \& Orlando, 2016) by removing adaptor sequences, sorting reads based

141 on tag combinations and filtering out stretches of low quality bases $(\mathrm{Q}<30)$. Additionally, reads

142 containing $>5$ low quality bases after trimming and reads with tags having $>2$ mismatches to

143 barcodes were removed. A complete overview of extraction- and sequencing batches, sample IDs 
144 and read names can be found in the supplementary material (Table S3). Technical replicates for

145 each sample were merged and primer sequences removed, allowing a maximum error rate of $15 \%$

146 using Cutadapt 2.10 (Martin, 2011). To ensure the same directionality of sequences (note that an

147 adapter-ligation based PCR-free library preparation was used), reads sequenced in the reverse-

148 forward direction were reversed based on primer location. All downstream filtering, analyses and

149 original illustrations were performed in Rstudio/v.1.2.5033 (RStudio Team, 2019). Reads were

150 filtered based on quality and specific error signatures error models of each respective round of

151 sequencing using DADA2 (Callahan et al., 2016). ASVs were inferred, forward and reverse reads

152 merged with a minimum overlap of $5 \mathrm{bp}$ and the taxonomy was assigned with the Silva/v132

153 taxonomic database (Quast et al., 2013) using DADA2. Finally the five datasets from each

154 respective sequencing rounds were merged for further post-clustering filtering and chimeric 155 sequences removed using DADA2. LULU (Frøslev et al., 2017) was used for post-clustering

156 removal of erroneous ASVs. To identify samples not reaching a diversity plateau due to

157 sequencing depth, rarefaction curves were plotted. Decontamination of potential contaminant

158 ASVs were done using Decontam (Davis et al., 2018) with the prevalence method and default

159 settings. This resulted in the removal of 19 contaminant ASVs with an average relative abundance

160 across species and sample types of $0.0001 \pm 0.0005$ (Table S4). All ASVs identified as belonging

161 to Vertebrates at phylum level or mitochondria at family level were removed, as well as all ASVs

162 mapping to Chloroplast at order level as these might originate from ingested plant material

163 (Hanshew et al., 2013). Next, the dataset was split into sub-datasets corresponding to each of the

164 three species. To remove the impact of low-abundance and low-prevalence microbes, only ASVs

165 present in $\geq 2$ samples and with a relative abundance $>0.1 \%$ in at least one sample were used in 166 downstream analyses. Phylogenetic trees used in the analysis of diversity and compositional 167 variation were generated by aligning ASVs using Clustal-Omega (Sievers et al., 2011) and then 168 RAxML-NG (Kozlov et al., 2019) were used to infer a maximum likelihood tree.

169 Statistical analysis

170 The following analyses were done using the R packages phyloseq (McMurdie \& Holmes, 2013), 171 ggpubr (Kassambara, 2020), hilldiv (Alberdi \& Gilbert, 2019), nlme (Pinheiro et al., 2021), stats 
172 (R Core Team, 2020), vegan (Oksanen et al., 2018) and Hmsc (Tikhonov et al., 2020). Functions

173 used in their respective packages will be referred to as 'package::function'.

\section{Analysis of diversity}

175 Paired diversity plots for each of the three species were generated using ggpubr::ggpaired. 176 Diversity analyses were done using the Hill numbers framework (Chao, Chiu \& Jost, 2014). The 177 diversity profiles for all locations across all three species were plotted using hilldiv::div_profile 178 (Fig. S1). The steep decline in the effective number of ASVs going from a q-value of 0 to 1 179 indicates a high level of unevenness between ASVs. Hence, a q-value of 1 was chosen for Hill180 numbers based downstream diversity analyses, so that ASVs were weighed according to their relative read abundances (Jost, 2006). Furthermore, to account for the phylogenetic relatedness

182 between ASVs, we also included the generated phylogenetic trees in the analysis. To satisfy the 183 assumptions of the linear mixed-effects model, two paired samples were visually identified as 184 outliers by plotting the residuals and subsequently removed from the AI data set (Fig. S2). 185 Diversity of individual samples was computed using hill_div::div_hill. A non-parametric Kruskal186 Wallis test was used to test for differences in diversity across fish species for each of the two 187 sample types followed by a post-hoc Dunn test with Benjamini-Hochberg correction as 188 implemented in hill_div::div_test. Linear mixed-effect models (nlme::lme) were used to examine 189 how sample type influences the diversity of individual fish gut microbiomes using the Restricted 190 Maximum Likelihood (REML) method for parameter estimation. Due to the natural 191 interdependency of paired intestinal wall-digesta samples and individual fish within locations,

192 Fish_ID was nested inside Locations and both were treated as random effects and Sample.type as 193 a fixed effect. Assumptions were verified by plotting the residuals.

\section{Compositional variation}

195 Compositional differences were measured by means of Jaccard-type overlap metric, including 196 phylogenetic relationships between ASVs (Chiu, Jost \& Chao, 2014; Chao et al., 2019; Alberdi \& 197 Gilbert, 2019) and computed using the function hill_div::pair_dis. Based on the generated 198 dissimilarity matrix, phyloseq::ordinate was used to create non-metric multidimensional scaling 199 (NMDS) ordinations for the three species and phyloseq::plot_ordination was used to generate 200 plots. To test the null hypothesis of no effect of sample type and location on bacterial community 
201 composition, a PERMANOVA analysis was done using vegan::adonis (Permanova 1) with the

202 above-described Jaccard-type dissimilarity matrix as response. Fish_ID was used as strata to 203 account for the dependency between intestinal wall-digesta samples within the same individual 204 and Sample.type and Location were used as fixed explanatory factors (including their interaction). 205 A second PERMANOVA (Permanova 2) was done to test whether the microbial community of 206 digesta samples was influenced more by environmental factors compared to the community of the 207 intestinal wall samples using Location as the fixed explanatory factor and either the Jaccard-type 208 dissimilarity table for intestinal wall or digesta samples as response. For both PERMANOVAs, 209999 permutations were used. The assumption of homogeneous dispersion in compared groups was

210 tested using vegan::betadisper and stats::anova. The assumption of homogeneity of within-group

211 dispersions was violated for locations for $\mathrm{VH}$ because of the presence of two individuals with very

212 distinct microbiomes. To ensure the robustness of our results, we repeated the analysis after 213 removing those fish with outlying values, which led to homogeneous dispersions and similar 214 results.

215

216 To identify microbial ASVs and families enriched in intestinal wall samples as compared to digesta 217 samples we analysed the data using a hierarchical modelling of species communities (HMSC) 218 framework (Tikhonov et al., 2020) as implemented in the R package Hmsc (Tikhonov et al., 2021). 219 HMSC is a class of joint species distribution models (Warton et al., 2015) to analyse multivariate 220 community data. For that, a hierarchical model is constructed in the generalised linear model 221 (GLM) framework and using Bayesian inference. We fitted models with two alternative response 222 data: 1) bacterial ASV sequence counts collected in each sample type in fish individuals of the 223 three species and, 2) bacterial families sequence counts, generated by adding up the sequences of 224 the ASVs belonging to each family. Additionally, the three fish species were analysed in three 225 independent models, thus making a total of six models. As the data were zero inflated, we applied 226 a hurdle model, similar to previous publications using amplicon sequencing data (Odriozola et al., 227 2021). This type of model consists of two parts, one modelling the presence-absence of species 228 and the other modelling abundance conditional on presence. To fit the first model we transformed 229 all non-zero values in the dataset to one, to create a presence-absence matrix. Then, for the second 230 model, we generated another dataset where we shifted all zeros to missing values, and kept all 231 nonzeros in their values. In the presence-absence part of the model, we applied a binomial model 
232 with probit link function to each ASV, whereas to model abundances conditional on presences

233 (scaled to mean zero and unit variance) we used the log-normal model. Then, the two components

234 of the model were fitted consecutively. See (Ovaskainen \& Abrego, 2020) for more details on how

235 to apply hurdle models in HMSC to model zero inflated data.

236

237 As fixed explanatory variables in the matrix X of HMSC we included the categorical variable 238 Sample.type, as well as the continuous variable log-transformed Sequencing.depth, which 239 controlled for the variation in sequencing depth among samples. To account for the nested study 240 design, we included Fish_ID and Location level random effects in the models. A significant 241 association with a specific sample type in the binomial model means that the ASV has a higher 242 probability of occurrence in that sample type. A significant association in the log-normal model 243 means that, when present, the ASV is more abundant in a sample type. A posterior probability of $244>0.7$ ( $<0.3$ for negative association) was considered as moderate statistical support for the 245 association with a sample type, whereas a posterior probability of $>0.9 \quad(<0.1$ for negative 246 association) was considered as strong support.

247

248

249

We fitted the models with Hmsc::Hmsc assuming the default priors and sampled the posterior distribution with Hmsc::sampleMcmc that ran four Marcov Chain Monte Carlo (MCMC) chains, each of which was run for 37,500 iterations, of which 12,500 were discarded as burn-in. We thinned by 100 to obtain a total of 250 posterior samples per chain and 1,000 posterior samples in total. We assessed MCMC convergence by measuring potential scale reduction factor (Tikhonov et al., 2020) for the beta parameters (measuring the response to sample type) (Fig. S3).

\section{Results}

255 Filtering, trimming and the removal of contaminant sequences resulted in a total of $18,882,099$ 256 merged reads. The removal of merged reads mapping to either Vertebrata (37,803 merged reads) 257 at phylum level, Chloroplast (849,196 merged reads) at order level and mitochondria at family 258 level $(175,284)$ resulted in $17,819,816$ remaining merged reads with all samples reaching diversity 259 saturation in sequencing depth (Fig. S4). The removal of outliers and low abundant ASVs resulted 
260 in 474 ASVs assigned to AI, $570 \mathrm{ASV}$ to GH and 114 ASVs to VH. A complete overview of post-

261 filtering taxonomy and abundances can be found in the supplemental material (Data S1).

262

263 Fish species exhibited different overall microbial diversities and communities (Kruskal-Wallis 264 test, Fig. 2a-b, Table 1). For both intestinal wall and digesta samples, post-hoc analysis revealed 265 that the diversity was significantly different between VH and the two other species, whereas no 266 significant differences were found between AI and GH (Fig. 2a). Regardless of the host species, 267 linear mixed effects models revealed that intestinal wall samples had a significantly lower diversity 268 compared to their corresponding digesta sample for AI, GH and VH with an average diversity 269 decline of $16 \%, 23 \%$ and $27 \%$ relative to the mean intercept for digesta samples, respectively (Fig. $2702 a)$.

271

272 Microbial composition was significantly different between intestinal wall and digesta samples for 273 all three species (Permanova 1, Table 1). Sample type (intestinal wall vs. digesta) was able to 274 explain $1-12 \%$ of the variance, however the majority of the explained variance (29-49\%) was 275 attributed to differences between locations (Permanova 1, Table 1). NMDS plots supported these 276 findings with samples from the same location clustering closer together and within locations paired 277 samples displaying smaller, but considerable distance between sample types (Fig. 2b). The NMDS

278 plot of the two sample types for all three species indicated that there were no general patterns in 279 which samples of the same type clustered closer together across species and that the extent of 280 differences between the two sample types were relative to the individual fish (Fig. 2c). We further 281 investigated whether the relative effect of location was greater on digesta samples relative to 282 intestinal wall samples and found location to have a significant effect on digesta samples for all 283 three species, explaining 27-68\% of the variance (Permanova 2, Table 1). Location also had a 284 significant effect on the composition of intestinal wall samples from AI and GH, but the amount 285 of explained variance was lower compared to digesta samples (17-48\%, all species; Permanova 2, 286 Table 1).

287

288 The majority of the families were negatively associated with the intestinal wall in both binomial $289(\mathrm{AI}=90.7 \%, \mathrm{GH}=86.4 \%$ and $\mathrm{VH}=100 \%)$ and log-normal $(\mathrm{AI}=81.4 \%, \mathrm{GH}=92.8 \%$ and $\mathrm{VH}=94.6 \%)$ 290 parts of the HMSC models in AI, GH and VH: this means that most families had higher probability 291 of occurrence and, when present, they were more abundant in digesta samples (Fig. 3, Fig. S5, 
292 Data S2). The same pattern was observed at ASV level (Data S2). Across the three species, the 293 log-normal part of the models identified six families $(\mathrm{AI}=4, \mathrm{GH}=0$ and $\mathrm{VH}=2)$ that, when they 294 occurred, were more abundant in intestinal wall samples with strong statistical support (posterior 295 probability $>0.9)$ and three families $(\mathrm{AI}=1, \mathrm{GH}=2$ and $\mathrm{VH}=0)$ with moderate statistical support 296 (posterior probability $>0.7$ ). Some of these families were enriched in more than one fish species 297 and in total they represented seven unique families. No families or ASVs were positively 298 associated with the intestinal wall in the binomial part of the model (Fig. S5, Data S2). At ASV 299 level, the log-normal part of the models found $25 \mathrm{ASVs}(\mathrm{AI}=8, \mathrm{GH}=10$ and $\mathrm{VH}=7)$ that were more 300 abundant in intestinal wall samples with strong statistical support and 58 ASVs $(\mathrm{AI}=21, \mathrm{GH}=34$ 301 and $\mathrm{VH}=3$ ) with moderate statistical support.

\section{Discussion}

303 Amplicon sequencing of paired intestinal wall and digesta samples from the GI tract of three small 304 cyprinodontiform fish species were able to identify two different microbial communities. We 305 found that intestinal wall samples from the three species were significantly less diverse than digesta samples and that the overall community composition is significantly different between the two kinds of samples for all three fish species. Furthermore, we identified seven families of bacteria to be significantly more abundant in intestinal wall samples across the three species.

Overall, AI and GH had gut microbial communities with similar levels of diversity, whereas VH exhibited lower diversity (Fig. 2a). Irrespective of the overall diversity, the intestinal wall microbiota was significantly less diverse compared to the paired digesta microbiota, which is in

312 concordance with previous studies comparing these two communities (Kim, Brunt \& Austin, 2007;

313 Gajardo et al., 2016; Nielsen et al., 2017; Riiser et al., 2018). The general higher diversity of 314 digesta samples is expected to be a result of the mixture of environmental microbes and food items

315 (Ringø et al., 2016; Legrand et al., 2020). We expected the intestinal wall community to also 316 include some of these environmental microbes, as we did not wash the intestinal wall or leave the 317 fish without access to food prior to sampling. Still, we observed a lower diversity in the intestinal 318 wall samples, which supports the hypothesis that it takes specialised traits for a microbe to adhere 319 and sustain a viable population in this environment (Nayak, 2010). 
321 The analysis of between sample type dissimilarity (Permanova 1, Table 1) supports that not only

322 are the intestinal wall community less diverse, but the community composition for all three species

323 is also significantly different to that of the digesta. Location explained most of the variance, with

324 substantial variation between species (Permanova 1, Table 1). Hence, we also separately examined

325 the effect of location on the two sample types and found that location explained a larger part of the

326 variation for digesta samples for all the included species compared to intestinal wall samples

327 (Permanova 2, Table 1). This is in line with what has previously been proposed about the transient

328 community being mainly influenced by environmental factors (Legrand et al., 2020) such as

329 temperature (Martin-Antonio et al., 2007; Kokou et al., 2018), salinity (Schmidt et al., 2015), level

330 of eutrophication (Restivo et al., 2021b), diets (Ringø et al., 2016) and the microbial communities

331 of the water column and sediments (Minich et al., 2020). Therefore, we warrant further research

332 into the influence of different environmental factors on both transient and resident communities as

333 well as the influence of intrinsic factors such as genotype (Kokou et al., 2018). Furthermore, the

334 large variation observed in the influence of location between the three species indicates that

335 microbial communities respond differently to the influence of environmental and intrinsic factors

336 between species of fish, which should also be the topic for further investigation.

337

338 Although the variation explained by the sample type was considerably lower than that explained

339 by location, we detected consistent patterns of microbial enrichment between the intestinal wall

340 and digesta across individuals and localities. A total of seven families were found to be more

341 abundant in the intestinal wall samples compared to the digesta samples (Fig. 3). Some of these

342 families have previously been found to include members closely associated with their host. For

343 instance, due to their small genome sizes, members of the family Mycoplasmataceae are suspected

344 to be intimately associated with their host and are often found to be dominating the gut microbiome

345 of Salmonids such as rainbow trout (Oncorhynchus mykiss), Atlantic salmon (Salmo salar) and

346 European whitefish (Coregonus lavaretus) (Brown, Wiens \& Salinas, 2019; Rasmussen et al.,

347 2021), although they also have been found to be dominating the GI tract in other fish species (Bano

348 et al., 2007; Burtseva et al., 2021). We found Mycoplasmataceae to be enriched in intestinal wall

349 samples from GH and VH, whereas in AI Mycoplasmataceae was enriched in the digesta sample.

350 Interestingly, we found that ASV_1 and ASV_6, both belonging to the genus Mycoplasma, were

351 highly enriched in intestinal wall samples of VH and GH, respectively (Data S2), but also that the 
352 same two ASVs were enriched in digesta samples from AI. This could suggest that members of

353 this family are present in the surrounding environment, but not all of them are able to colonise the 354 intestinal wall of all fish species. To further investigate this, future studies looking into microbial 355 differences between intestinal wall and digesta samples should ideally include water samples 356 collected from the different locations. A similar situation was observed for the families 357 Brevinemataceae and Desulfovibrionaceae. Brevinemataceae have been identified in the GI tract 358 of several fish species (Brown, Wiens \& Salinas, 2019; Uren Webster et al., 2020; Iwatsuki et al., 359 2021) and Desulfovibrionaceae in particular have earlier been found to be enriched in samples 360 from the intestinal wall compared to paired content samples from rabbitfish (Siganus fuscescens)

361 (Nielsen et al., 2017). The family Microbacteriaceae has previously been identified in relation to 362 fish (Boutin et al., 2012; Larios-Soriano et al., 2021), but as members of this family have been 363 found on the skin and in gut digesta it seems most likely that these are transient microbes. As with 364 Mycoplasmataceae, the small genome size of members in the family Saccharimonadaceae, 365 enriched in AI, have led researchers to propose a symbiotic lifestyle (Lemos et al., 2019) and as a 366 consequence we can not rule out a symbiotic relationship between members of Saccharimonadaceae and AI. The two remaining enriched families, A4b and Methylococcaceae, have to our knowledge not been identified in association with a fish host, but rather to plant root microbiomes (Vik et al., 2013; Barelli et al., 2020) and from environmental samples (Taubert et al., 2019), respectively. Even though the diversity and composition of the microbial communities of intestinal wall and digesta samples for GH were significantly different, we were unable to identify families with strong statistical support to have a higher abundance in intestinal wall samples. This could be attributed to the fragility of the GI tract of GH compared to the two other species, making it hard to squeeze out digesta without fragmenting the intestine, which could have resulted in a higher proportion of digesta left in the intestinal wall samples.

Identifying whether the members of the enriched families are truly a part of the resident microbial community in these fish will need further investigations using more detailed methods. These methods includes shotgun sequencing, which would allow researchers to look for genes within each respective metagenome assembled genome (MAG) related to host adaptations, but this method can be hampered by the large proportion of host DNA found in intestinal wall samples 
383 the level of replication of MAGs within a metagenome, which could be used as a proxy for resident

384 time in GI tract, assuming that resident microbes will have a higher replication rate at a any given

385 time point compared to transient microbes (Brown et al., 2016). Other alternative methods such as

386 fluorescence in situ hybridisation (Shi, Grodner \& De Vlaminck, 2021), electron microscopy

387 (Ringø et al., 2003) and laser capture microdissection (Riva et al., 2019) can provide researchers

388 with the spatial resolution needed to establish the level of intimacy between microbial cells and

389 host cells.

390

391 Even though we in this study were able to find members of the microbiome that are enriched in

392 intestinal wall samples, we are conscious that the simple sampling procedure applied in the current

393 study is prone to yield a substantial level of spillover between paired digesta and intestinal wall

394 samples. As a consequence we do not expect to find many families/ASVs that have a higher chance

395 of being found in the intestinal wall compared to digesta (presence/absence) as we expect most of

396 the spillover to originate from this sample type. Therefore, we mainly relied on the abundance data

397 as we expected the signal to be stronger than for the presence-absence data. We observed a number

398 of enriched families/ASVs in the intestinal wall samples that we find unlikely to be part of the

399 resident microbial community based on previous studies on these taxa. Hence, the results should

400 not be trusted blindly. We are aware that many of the microbes that are present in the intestinal

401 wall samples, but not identified to be significantly more frequently found or abundant in intestinal

402 wall samples by the HMSC models, could in fact be a part of the resident microbial community.

403 With that in mind, it is most probable that the enrichment analysis would be able to identify more

404 microbes to be a part of the resident microbiota if further rinsing of the empty intestine were done,

405 but for small fish this is not always possible.

406

407 Given the nature of gut microbiome samples collected from small fish it is difficult to obtain 408 samples that only capture microbes from either the resident or transient microbial communities.

409 Both communities can be of relevance to answer questions regarding the overall role of the 410 microbial gut community, but in some cases the goal is to determine which microbes can be 411 considered as being adapted to and potentially essential for the fish host. This could for instance

412 be the case in captive breeding of small or early life stages of endangered fish species (Lavoie et 413 al., 2018; West et al., 2019; Abdul Razak \& Scribner, 2020) or to increase the survival rate of early 
414 life stages of commercially important aquaculture species (Abdul Razak \& Scribner, 2020; Borges 415 et al., 2021). We acknowledge that due to resource limitation, in many cases it will not be possible 416 to apply more detailed methods to identify resident microbes. By using a combination of relatively 417 cost-efficient amplicon sequencing and conservative statistical modelling we believe it is possible 418 to make meaningful inference regarding the origin of a given taxa, as long as results are compared 419 and evaluated based on the findings of other studies. When it is not possible to model enrichment 420 between paired samples, for instance when only one kind of sample is collected, we encourage 421 researchers to be conscious of whether a given sampling procedure is likely to capture the resident 422 or the transient community, or a mixture of the two, when reporting the results from these studies.

\section{Conclusion}

424 In this study we found that paired samples from the intestinal wall and digesta coming from three 425 small fish species harbor different microbial communities. The diversity was significantly lower 426 in samples from the intestinal wall for the three fish species and the community composition was 427 significantly different between sample types for all three species. Using hierarchical modelling of 428 species communities we identified a total of 8 unique families to be significantly enriched in 429 intestinal wall samples. Based on these findings we conclude that the sampling procedure used in 430 the current study combined with conservative statistical modelling is capable of identifying host431 associated microbes, but these results should be critically evaluated and that more comprehensive 432 methods should be used to further examine the true level of host-association. When collection of 433 paired samples is not possible, we encourage researchers to be aware that different sampling 434 protocols are likely to capture different parts of the fish gut microbiome and that this should be 435 incorporated in the interpretation of the results. 


\section{Supplemental information}

\section{Additional information and declarations}

\section{Author contributions}

439 Conceptualization: L.N. and A.A.; Sample collection: L.N., A.A., G.M. and O.A; Writing -

440 original draft: L.N.; Writing - Review and editing: All authors; Data analysis and visualisation:

441 L.N. I.O. and O.A.; Funding Acquisition: A.A..

\section{Data availability}

443 Demultiplexed reads are available at the ENA repository under accession number PRJEB48573.

444 Note that_M and_D indicate intestinal wall and digesta samples, respectively. Code can be 445 found at: https://github.com/LasseNyholm/Gut-microbiota-differences-between-paired-mucus446 and-digesta-samples-in-three-small-species-of-fish

\section{Acknowledgement}

448 The authors would like to thank the staff at Centro de Conservación de Especies Dulceacuícolas 449 de la Comunitat Valenciana, and especially to Pilar Risueño and Jesús Hernández, for their 450 indispensable help in collecting samples from the three fish species as well as providing knowledge 451 on the biology of the species. O.A. and A.A. acknowledge the Danish National Research 452 Foundation grant DNRF143. A.A. further acknowledges the Lundbeckfonden grant R250-20174531351.

454

455 References

456 Abdul Razak S, Scribner KT. 2020. Ecological and Ontogenetic Components of Larval Lake 
457

458

459

460

461

462

463

464

465

466

467

468

469

470

471

472

473

474

475

476

477

478

479

Sturgeon Gut Microbiota Assembly, Successional Dynamics, and Ecological Evaluation Of Neutral Community Processes. Applied and environmental microbiology. DOI: 10.1128/AEM.02662-19.

Aizpurua O, Nyholm L, Morris E, Chaverri G, Herrera Montalvo LG, Flores-Martinez JJ, Lin A, Razgour O, Gilbert MTP, Alberdi A. 2021. The role of the gut microbiota in the dietary niche expansion of fishing bats. Animal microbiome 3:76.

Alberdi A, Aizpurua O, Gilbert MTP, Bohmann K. 2017. Scrutinizing key steps for reliable metabarcoding of environmental samples. Methods in ecology and evolution / British Ecological Society 17:730.

Alberdi A, Gilbert MTP. 2019. A guide to the application of Hill numbers to DNA-based diversity analyses. Molecular ecology resources 19:804-817.

Almeida AR, Domingues I, Henriques I. 2021. Zebrafish and water microbiome recovery after oxytetracycline exposure. Environmental pollution 272:116371.

Bano N, DeRae Smith A, Bennett W, Vasquez L, Hollibaugh JT. 2007. Dominance of Mycoplasma in the guts of the Long-Jawed Mudsucker, Gillichthys mirabilis, from five California salt marshes. Environmental microbiology 9:2636-2641.

Barelli L, Waller AS, Behie SW, Bidochka MJ. 2020. Plant microbiome analysis after Metarhizium amendment reveals increases in abundance of plant growth-promoting organisms and maintenance of disease-suppressive soil. PloS one 15:e0231150.

Barko PC, McMichael MA, Swanson KS, Williams DA. 2018. The Gastrointestinal Microbiome: A Review. Journal of veterinary internal medicine / American College of Veterinary Internal Medicine 32:9-25.

Binladen J, Gilbert MTP, Bollback JP, Panitz F, Bendixen C, Nielsen R, Willerslev E. 2007. The 
use of coded PCR primers enables high-throughput sequencing of multiple homolog amplification products by 454 parallel sequencing. PloS one 2:e197.

482

483

484

Bolnick DI, Snowberg LK, Hirsch PE, Lauber CL, Org E, Parks B, Lusis AJ, Knight R, Caporaso JG, Svanbäck R. 2014. Individual diet has sex-dependent effects on vertebrate gut microbiota. Nature communications 5:4500.

Borges N, Keller-Costa T, Sanches-Fernandes GMM, Louvado A, Gomes NCM, Costa R. 2021. Bacteriome Structure, Function, and Probiotics in Fish Larviculture: The Good, the Bad, and the Gaps. Annual review of animal biosciences 9:423-452.

Boutin S, Bernatchez L, Audet C, Derôme N. 2012. Antagonistic effect of indigenous skin bacteria of brook charr (Salvelinus fontinalis) against Flavobacterium columnare and F. psychrophilum. Veterinary microbiology 155:355-361.

Breen P, Winters AD, Nag D, Ahmad MM, Theis KR, Withey JH. 2019. Internal Versus External Pressures: Effect of Housing Systems on the Zebrafish Microbiome. Zebrafish $16: 388-400$.

Brown CT, Olm MR, Thomas BC, Banfield JF. 2016. Measurement of bacterial replication rates in microbial communities. Nature biotechnology 34:1256-1263.

Brown RM, Wiens GD, Salinas I. 2019. Analysis of the gut and gill microbiome of resistant and susceptible lines of rainbow trout (Oncorhynchus mykiss). Fish \& shellfish immunology $86: 497-506$.

Burns AR, Miller E, Agarwal M, Rolig AS, Milligan-Myhre K, Seredick S, Guillemin K, Bohannan BJM. 2017. Interhost dispersal alters microbiome assembly and can overwhelm host innate immunity in an experimental zebrafish model. Proceedings of the National Academy of Sciences of the United States of America 114:11181-11186. 
503 Burtseva O, Kublanovskaya A, Fedorenko T, Lobakova E, Chekanov K. 2021. Gut microbiome 504 of the White Sea fish revealed by 16S rRNA metabarcoding. Aquaculture 533:736175.

505 Callahan BJ, McMurdie PJ, Rosen MJ, Han AW, Johnson AJA, Holmes SP. 2016. DADA2:

506 High-resolution sample inference from Illumina amplicon data. Nature methods 13:581$507 \quad 583$.

508 Caporaso JG, Lauber CL, Walters WA, Berg-Lyons D, Lozupone CA, Turnbaugh PJ, Fierer N, 509 Knight R. 2011. Global patterns of 16S rRNA diversity at a depth of millions of sequences 510 per sample. Proceedings of the National Academy of Sciences of the United States of $511 \quad$ America 108 Suppl 1:4516-4522.

512 Carøe C, Bohmann K. 2020. Tagsteady: a metabarcoding library preparation protocol to avoid

513 false assignment of sequences to samples. DOI: 10.1101/2020.01.22.915009.

514 Chao A, Chiu C-H, Jost L. 2014. Unifying species diversity, phylogenetic diversity, functional

515 diversity, and related similarity and differentiation measures through Hill Numbers. Annual 516 review of ecology, evolution, and systematics 45:297-324.

517 Chao A, Chiu C-H, Villéger S, Sun I-F, Thorn S, Lin Y-C, Chiang J-M, Sherwin WB. 2019. An

518 attribute-diversity approach to functional diversity, functional beta diversity, and related 519 (dis)similarity measures. Ecological monographs 89:e01343.

520 Chiu C-H, Jost L, Chao A. 2014. Phylogenetic beta diversity, similarity, and differentiation 521 measures based on Hill numbers. Ecological monographs 84:21-44.

522 Compant S, Samad A, Faist H, Sessitsch A. 2019. A review on the plant microbiome: Ecology, 523 functions, and emerging trends in microbial application. Journal of advertising research $524 \quad 19: 29-37$.

525 Davis NM, Proctor DM, Holmes SP, Relman DA, Callahan BJ. 2018. Simple statistical 
526 identification and removal of contaminant sequences in marker-gene and metagenomics 527 data. Microbiome 6:226.

528 DeAngelis MM, Wang DG, Hawkins TL. 1995. Solid-phase reversible immobilization for the 529 isolation of PCR products. Nucleic acids research 23:4742-4743.

530 Feng J-B, Hu C-Q, Luo P, Zhang L-P, Chen C. 2010. Microbiota of yellow grouper (Epinephelus $531 \quad$ awoora Temminck \& Schlegel, 1842) fed two different diets. Aquaculture research 41:1778-1790.

533 Frøslev TG, Kjøller R, Bruun HH, Ejrnæs R, Brunbjerg AK, Pietroni C, Hansen AJ. 2017.

534 Algorithm for post-clustering curation of DNA amplicon data yields reliable biodiversity $535 \quad$ estimates. Nature communications 8:1188.

536 Gajardo K, Rodiles A, Kortner TM, Krogdahl Å, Bakke AM, Merrifield DL, Sørum H. 2016. A 537 high-resolution map of the gut microbiota in Atlantic salmon (Salmo salar): A basis for $538 \quad$ comparative gut microbial research. Scientific reports 6:30893.

539 Hanshew AS, Mason CJ, Raffa KF, Currie CR. 2013. Minimization of chloroplast contamination 540 in 16S rRNA gene pyrosequencing of insect herbivore bacterial communities. Journal of $541 \quad$ microbiological methods 95:149-155.

542 Iwatsuki T, Kanazawa T, Ogasawara T, Hosotani K, Tsuchiya K, Watanabe S, Suzuki T,

543 Moriuchi R, Kanesaki Y, Dohra H. 2021. 16S rRNA Gene Amplicon Sequencing of Gut

544 Microbiota in Three Species of Deep-Sea Fish in Suruga Bay, Japan. Microbiology resource 545 announcements 10. DOI: 10.1128/MRA.01260-20.

546 Jost L. 2006. Entropy and diversity. Oikos 113:363-375.

547 Kassambara A. 2020. “ggplot2” Based Publication Ready Plots.

548 Kim D-H, Brunt J, Austin B. 2007. Microbial diversity of intestinal contents and mucus in 

rainbow trout (Oncorhynchus mykiss). Journal of applied microbiology 102:1654-1664.

550 Kohl KD, Amaya J, Passement CA, Dearing MD, McCue MD. 2014. Unique and shared

551 responses of the gut microbiota to prolonged fasting: a comparative study across five

552 classes of vertebrate hosts. FEMS microbiology ecology 90:883-894.

553 Kokou F, Sasson G, Nitzan T, Doron-Faigenboim A, Harpaz S, Cnaani A, Mizrahi I. 2018. Host

554 genetic selection for cold tolerance shapes microbiome composition and modulates its

555 response to temperature. eLife 7. DOI: 10.7554/eLife.36398.

556 Kozlov AM, Darriba D, Flouri T, Morel B, Stamatakis A. 2019. RAxML-NG: a fast, scalable

557 and user-friendly tool for maximum likelihood phylogenetic inference. Bioinformatics $558 \quad 35: 4453-4455$.

559 Lan C-C, Love DR. 2012. Molecular Characterisation of Bacterial Community Structure along 560 the Intestinal Tract of Zebrafish (Danio rerio): A Pilot Study. ISRN microbiology $561 \quad 2012: 590385$.

562 Larios-Soriano E, Re-Araujo AD, Gómez-Gil B, Tovar Ramírez D, Trejo-Escamilla I, Galaviz 563 MA. 2021. Reciprocal effect of temperature and dietary lipids on metabolic performance 564 and gut microbiota of Yellowtail kingfish ( Seriola lalandi ) juveniles. Aquaculture $565 \quad$ research. DOI: 10.1111/are.15480.

566 Lavoie C, Courcelle M, Redivo B, Derome N. 2018. Structural and compositional mismatch 567 between captive and wild Atlantic salmon (Salmo salar) parrs' gut microbiota highlights the 568 relevance of integrating molecular ecology for management and conservation methods.

$569 \quad$ Evolutionary applications 11:1671-1685.

570 Legrand TPRA, Wynne JW, Weyrich LS, Oxley APA. 2020. A microbial sea of possibilities:

571 current knowledge and prospects for an improved understanding of the fish microbiome. 
Reviews in Aquaculture 12:1101-1134.

573 Lemos LN, Medeiros JD, Dini-Andreote F, Fernandes GR, Varani AM, Oliveira G, Pylro VS.

574 2019. Genomic signatures and co-occurrence patterns of the ultra-small Saccharimonadia

575 (phylum CPR/Patescibacteria) suggest a symbiotic lifestyle. Molecular ecology 28:4259-

$576 \quad 4271$.

577 Li T, Qi M, Gatesoupe F-J, Tian D, Jin W, Li J, Lin Q, Wu S, Li H. 2019. Adaptation to Fasting 578 in Crucian Carp (Carassius auratus): Gut Microbiota and Its Correlative Relationship with 579 Immune Function. Microbial ecology 78:6-19.

580 Llewellyn MS, Boutin S, Hoseinifar SH, Derome N. 2014. Teleost microbiomes: the state of the 581 art in their characterization, manipulation and importance in aquaculture and fisheries.

$582 \quad$ Frontiers in microbiology 5:207.

583 Marotz CA, Sanders JG, Zuniga C, Zaramela LS, Knight R, Zengler K. 2018. Improving saliva 584 shotgun metagenomics by chemical host DNA depletion. Microbiome 6:42.

585 Martin M. 2011. Cutadapt removes adapter sequences from high-throughput sequencing reads. $586 \quad$ EMBnet.journal 17:10-12.

587 Martin-Antonio B, Manchado M, Infante C, Zerolo R, Labella A, Alonso C, Borrego JJ. 2007.

588 Intestinal microbiota variation in Senegalese sole (Solea senegalensis) under different

$589 \quad$ feeding regimes. Aquaculture research 38:1213-1222.

590 McMurdie PJ, Holmes S. 2013. phyloseq: an R package for reproducible interactive analysis and 591 graphics of microbiome census data. PloS one 8:e61217.

592 Mekuchi M, Asakura T, Sakata K, Yamaguchi T, Teruya K, Kikuchi J. 2018. Intestinal

593 microbiota composition is altered according to nutritional biorhythms in the leopard coral 594 grouper (Plectropomus leopardus). PloS one 13:e0197256. 
595 Minich JJ, Poore GD, Jantawongsri K, Johnston C, Bowie K, Bowman J, Knight R, Nowak B, 596 Allen EE. 2020. Microbial Ecology of Atlantic Salmon (Salmo salar) Hatcheries: Impacts of 597 the Built Environment on Fish Mucosal Microbiota. Applied and environmental 598 microbiology 86. DOI: 10.1128/AEM.00411-20.

599 Muyzer G, de Waal EC, Uitierlinden AG. 1993. Reaction-Amplified Genes Coding for 16S $600 \quad$ rRNA. Applied and Environmental Microbiology 59:695-700.

601 Nayak SK. 2010. Role of gastrointestinal microbiota in fish. Aquaculture research 41:1553$602 \quad 1573$.

603 Nielsen S, Wilkes Walburn J, Vergés A, Thomas T, Egan S. 2017. Microbiome patterns across 604 the gastrointestinal tract of the rabbitfish Siganus fuscescens. PeerJ 5:e3317.

605 Nyholm L, Koziol A, Marcos S, Botnen AB, Aizpurua O, Gopalakrishnan S, Limborg MT, 606 Gilbert MTP, Alberdi A. 2020. Holo-omics: integrated host-microbiota multi-omics for 607 basic and applied biological research. iScience:101414.

608 Odriozola I, Abrego N, Tláskal V, Zrůstová P, Morais D, Větrovský T, Ovaskainen O, Baldrian 609 P. 2021. Fungal Communities Are Important Determinants of Bacterial Community 610 Composition in Deadwood. mSystems 6. DOI: 10.1128/mSystems.01017-20.

611 Oksanen J, Blanchet GF, Friendly M, Kindt R, Legendre P, McGlinn D, Minchin PR, O’Hara

612 RB, Simpson GL, Solymos P, Stevens MHH, Szoecs E, Wagner H. 2018. vegan:

613 Community Ecology Package.

614 Ovaskainen O, Abrego N. 2020. Joint Species Distribution Modelling: With Applications in R. 615 Cambridge University Press.

616 Pinheiro J, Bates D, DebRoy S, Sarkar D, R Core Team. 2021. nlme: Linear and Nonlinear 617 Mixed Effects Models. 
618 Quast C, Pruesse E, Yilmaz P, Gerken J, Schweer T, Yarza P, Peplies J, Glöckner FO. 2013. The

619 SILVA ribosomal RNA gene database project: improved data processing and web-based $620 \quad$ tools. Nucleic acids research 41:D590-6.

621 Quince C, Walker AW, Simpson JT, Loman NJ, Segata N. 2017. Shotgun metagenomics, from 622 sampling to analysis. Nature biotechnology 35:833-844.

623 Rasmussen JA, Villumsen KR, Duchêne DA, Puetz LC, Delmont TO, Sveier H, Jørgensen L von 624 G, Præbel K, Martin MD, Bojesen AM, Gilbert MTP, Kristiansen K, Limborg MT. 2021. 625 Genome-resolved metagenomics suggests a mutualistic relationship between Mycoplasma 626 and salmonid hosts. Communications biology 4:579.

627 R Core Team. 2020. R: A Language and Environment for Statistical Computing.

628 Restivo VE, Kidd KA, Surette MG, Bucking C, Wilson JY. 2021a. The gut content microbiome 629 of wild-caught rainbow darter is altered during laboratory acclimation. Comparative $630 \quad$ biochemistry and physiology. Part D, Genomics \& proteomics 39:100835.

631 Restivo VE, Kidd KA, Surette MG, Servos MR, Wilson JY. 2021b. Rainbow darter (Etheostoma 632 caeruleum) from a river impacted by municipal wastewater effluents have altered gut 633 content microbiomes. The Science of the total environment 751:141724.

634 Riiser ES, Haverkamp THA, Borgan Ø, Jakobsen KS, Jentoft S, Star B. 2018. A Single

635 Vibrionales 16S rRNA Oligotype Dominates the Intestinal Microbiome in Two 636 Geographically Separated Atlantic cod Populations. Frontiers in microbiology 9:1561.

637 Ringø E, Olsen RE, Mayhew TM, Myklebust R. 2003. Electron microscopy of the intestinal 638 microflora of fish. Aquaculture 227:395-415.

639 Ringø E, Zhou Z, Vecino JLG, Wadsworth S, Romero J, Krogdahl Å, Olsen RE, Dimitroglou A, 640 Foey A, Davies S, Owen M, Lauzon HL, Martinsen LL, De Schryver P, Bossier P, Sperstad 
641

642

643

644

645

646

647

648

649

650

651

652

653

654

655

656

657

658

659

660

661

662

663

S, Merrifield DL. 2016. Effect of dietary components on the gut microbiota of aquatic animals. A never-ending story? Aquaculture Nutrition 22:219-282.

Riva A, Kuzyk O, Forsberg E, Siuzdak G, Pfann C, Herbold C, Daims H, Loy A, Warth B, Berry D. 2019. A fiber-deprived diet disturbs the fine-scale spatial architecture of the murine colon microbiome. Nature communications 10:4366.

Rohland N, Reich D. 2012. Cost-effective, high-throughput DNA sequencing libraries for multiplexed target capture. Genome research 22:939-946.

RStudio Team. 2019. RStudio: Integrated Development for $R$.

Schmidt VT, Smith KF, Melvin DW, Amaral-Zettler LA. 2015. Community assembly of a euryhaline fish microbiome during salinity acclimation. Molecular ecology 24:2537-2550.

Schubert M, Lindgreen S, Orlando L. 2016. AdapterRemoval v2: rapid adapter trimming, identification, and read merging. BMC research notes 9:88.

Shi H, Grodner B, De Vlaminck I. 2021. Recent advances in tools to map the microbiome. Current opinion in biomedical engineering 19. DOI: 10.1016/j.cobme.2021.100289.

Sievers F, Wilm A, Dineen D, Gibson TJ, Karplus K, Li W, Lopez R, McWilliam H, Remmert M, Söding J, Thompson JD, Higgins DG. 2011. Fast, scalable generation of high-quality protein multiple sequence alignments using Clustal Omega. Molecular systems biology $7: 539$

Sullam KE, Essinger SD, Lozupone CA, O’Connor MP, Rosen GL, Knight R, Kilham SS, Russell JA. 2012. Environmental and ecological factors that shape the gut bacterial communities of fish: a meta-analysis. Molecular ecology 21:3363-3378.

Taubert M, Grob C, Crombie A, Howat AM, Burns OJ, Weber M, Lott C, Kaster A-K, Vollmers J, Jehmlich N, von Bergen M, Chen Y, Murrell JC. 2019. Communal metabolism by 
664

665

666

667

668

669

670

671

672

673

674

675

676

677

678

679

680

681

682

683

684

685

Methylococcaceae and Methylophilaceae is driving rapid aerobic methane oxidation in sediments of a shallow seep near Elba, Italy. Environmental microbiology 21:3780-3795.

Tikhonov G, Opedal ØH, Abrego N, Lehikoinen A, de Jonge MMJ, Oksanen J, Ovaskainen O. 2020. Joint species distribution modelling with the r-package Hmsc. Methods in ecology and evolution / British Ecological Society 11:442-447.

Tikhonov G, Ovaskainen O, Oksanen J, de Jonge M, Opedal O, Dallas T. 2021. Hmsc: Hierarchical Model of Species Communities.

Uren Webster TM, Rodriguez-Barreto D, Castaldo G, Gough P, Consuegra S, Garcia de Leaniz C. 2020. Environmental plasticity and colonisation history in the Atlantic salmon microbiome: A translocation experiment. Molecular ecology 29:886-898.

Vik U, Logares R, Blaalid R, Halvorsen R, Carlsen T, Bakke I, Kolstø A-B, Økstad OA, Kauserud H. 2013. Different bacterial communities in ectomycorrhizae and surrounding soil. Scientific reports 3:3471.

Warton DI, Blanchet FG, O’Hara RB, Ovaskainen O, Taskinen S, Walker SC, Hui FKC. 2015. So Many Variables: Joint Modeling in Community Ecology. Trends in ecology \& evolution 30:766-779.

West AG, Waite DW, Deines P, Bourne DG, Digby A, McKenzie VJ, Taylor MW. 2019. The microbiome in threatened species conservation. Biological conservation 229:85-98.

Wu S, Gao T, Zheng Y, Wang W, Cheng Y, Wang G. 2010. Microbial diversity of intestinal contents and mucus in yellow catfish (Pelteobagrus fulvidraco). Aquaculture 303:1-7.

Xia JH, Lin G, Fu GH, Wan ZY, Lee M, Wang L, Liu XJ, Yue GH. 2014. The intestinal microbiome of fish under starvation. BMC genomics 15:266.

Peer) reviewing PDF | (2021:11:67438:1:2:NEW 13 Jan 2022) 


\section{Table 1 (on next page)}

\section{Results from PERMANOVA.}

The R2 value indicates how much of the variance is explained by a given covariate and level of significance with $* *(p<0.01)$ and $* * *(p<0.001)$. Sample type:Location is the interaction between the two fixed explanatory factors. 


\begin{tabular}{|l|l|l|l|l|l|l|}
\hline \multirow{2}{*}{ Permanova 1 } & \multicolumn{2}{|l|}{ Aphanius iberus } & \multicolumn{2}{l|}{ Gambusia holbrooki } & \multicolumn{2}{l|}{ Valencia hispanica } \\
\cline { 2 - 7 } & $\mathrm{R} 2$ & $\mathrm{p}$-value & $\mathrm{R} 2$ & $\mathrm{p}$-value & $\mathrm{R} 2$ & $\mathrm{p}$-value \\
\hline Sample type & 0.081 & $<0.001^{* * *}$ & 0.017 & $<0.001^{* * *}$ & 0.120 & $<0.001^{* * *}$ \\
\hline Location & 0.497 & $<0.001^{* * *}$ & 0.486 & $0.005^{* *}$ & 0.292 & $<0.001^{* * *}$ \\
\hline Sample type:Location & 0.013 & 0.345 & 0.018 & 0.055 & 0.012 & 0.338 \\
\hline \multirow{3}{*}{ Permanova 2 } & Aphanius iberus & Gambusia holbrooki & Valencia hispanica \\
\cline { 2 - 7 } & $\mathrm{R} 2$ & $\mathrm{p}$-value & $\mathrm{R} 2$ & $\mathrm{p}$-value & $\mathrm{R} 2$ & $\mathrm{p}$-value \\
\hline Location (intestinal wall) & 0.485 & $<0.001^{* * *}$ & 0.454 & $<0.001^{* * *}$ & 0.151 & 0.097 \\
\hline Location (digesta) & 0.649 & $<0.001^{* * *}$ & 0.573 & $<0.001^{* * *}$ & 0.252 & $<0.001^{* * *}$ \\
\hline
\end{tabular}


Figure 1

Overview of sample types

The two sample types used in the study are denoted either by a capital W (intestinal wall) or

D (digesta) and an overview of the number of species, locations, samples, length and weight.

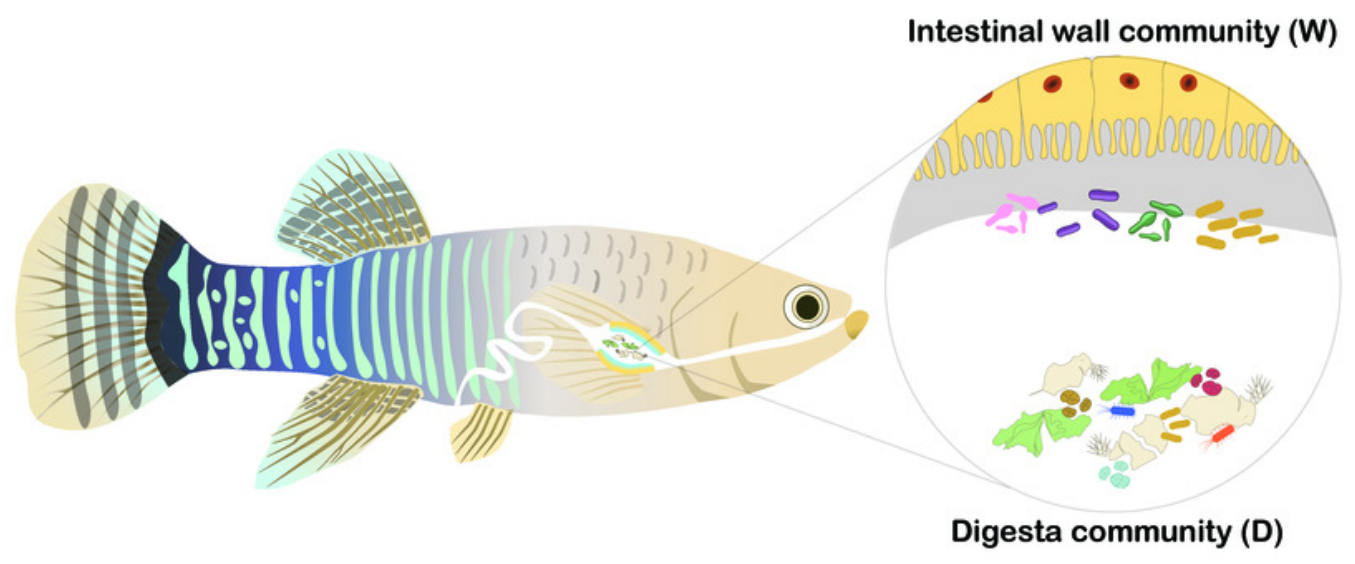

\begin{tabular}{c|c|c|c|c|c|c} 
Species & Locations & $\begin{array}{c}\text { Intestinal } \\
\text { wall } \\
\text { samples }\end{array}$ & $\begin{array}{c}\text { Digesta } \\
\text { samples }\end{array}$ & $\begin{array}{c}\text { Total } \\
\text { samples }\end{array}$ & $\begin{array}{c}\text { Average total } \\
\text { length (mm) }\end{array}$ & $\begin{array}{c}\text { Average } \\
\text { weight(g) }\end{array}$ \\
Aphanius iberus (AI) & 4 & 27 & 27 & 54 & $31.6 \pm 5.7$ & $0.52 \pm 0.27$ \\
\hline Gambusia holbrooki $(\mathrm{GH})$ & 4 & 26 & 26 & 52 & $36.3 \pm 7.5$ & $0.60 \pm 0.32$ \\
\hline Valencia hispanica $(\mathrm{VH})$ & 3 & 21 & 21 & 46 & $47.5 \pm 7.2$ & $1.54 \pm 0.70$
\end{tabular}




\section{Figure 2}

Overview of effective number of ASVs and NMDS plots of dissimilarities.

a) Boxplots showing the diversity of paired samples for each of the three species across all locations. Green lines indicate a decrease in diversity going from digesta samples to intestinal wall samples and red lines indicate an increase in diversity. Results from KruskalWallis test for overall differences in diversity across species and the post-hoc Dunn tests are shown above the boxplots and results from the linear mixed-effects models (Ime) are shown within each boxplot. The mean intercept refers to that of digesta samples, and the numbers over the black dotted lines are the mean slope values going from from digesta samples to their corresponding intestinal wall samples. b) NMDS plots of dissimilarity between samples within species and c) dissimilarity between samples across all species. Sample pairs are connected by lines, and the two types of samples are shown as either circles (digesta) or triangles (intestinal wall) and different locations are indicated by colour. 
Aphanius iberus

a) Kruskal-Wallis test:

Digesta: $p<0.001^{* * *}$
Intestinal wall: $p<0.001^{* * *}$

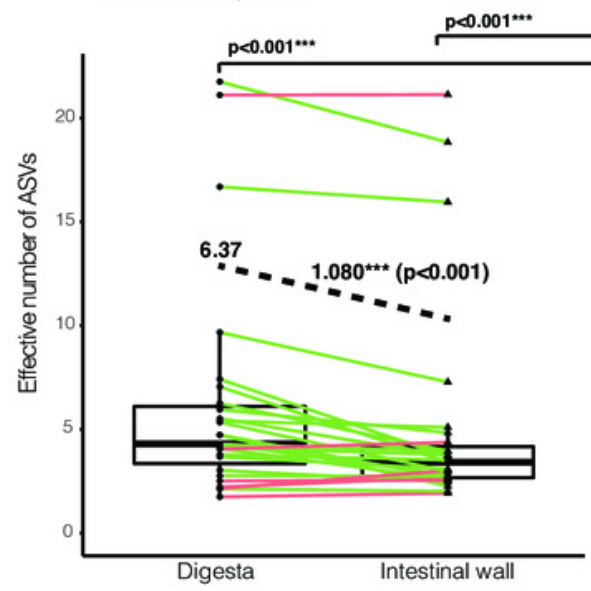

b)
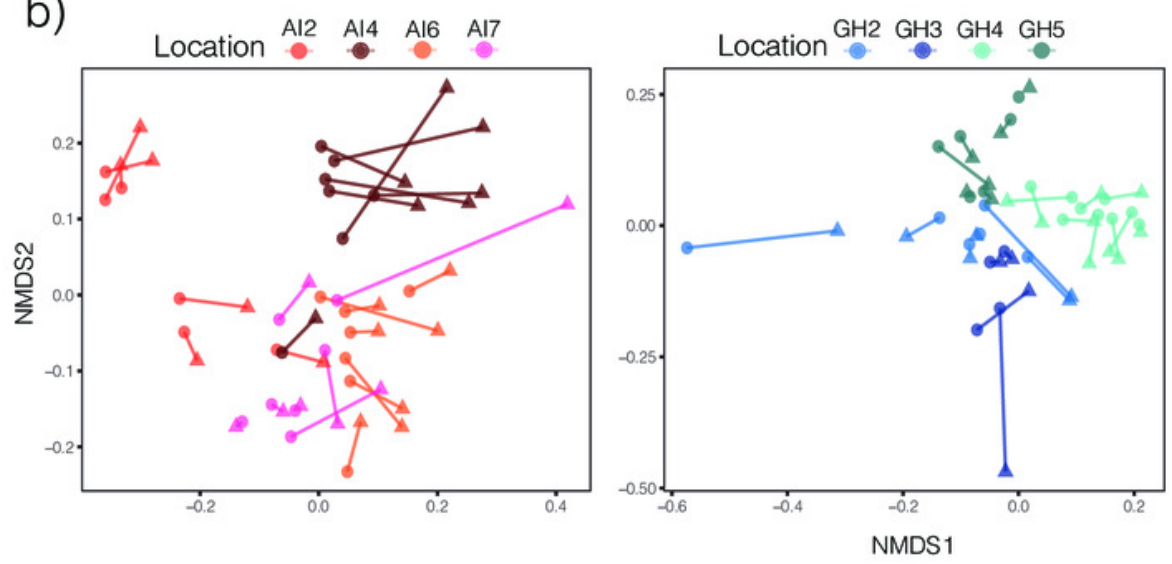

Mean intercept: 5.007 Slope: $-1.200^{*}(p=0.046)$
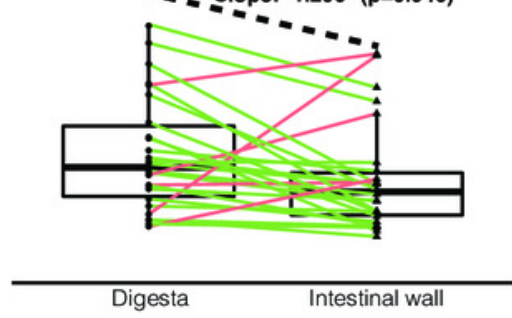

c)

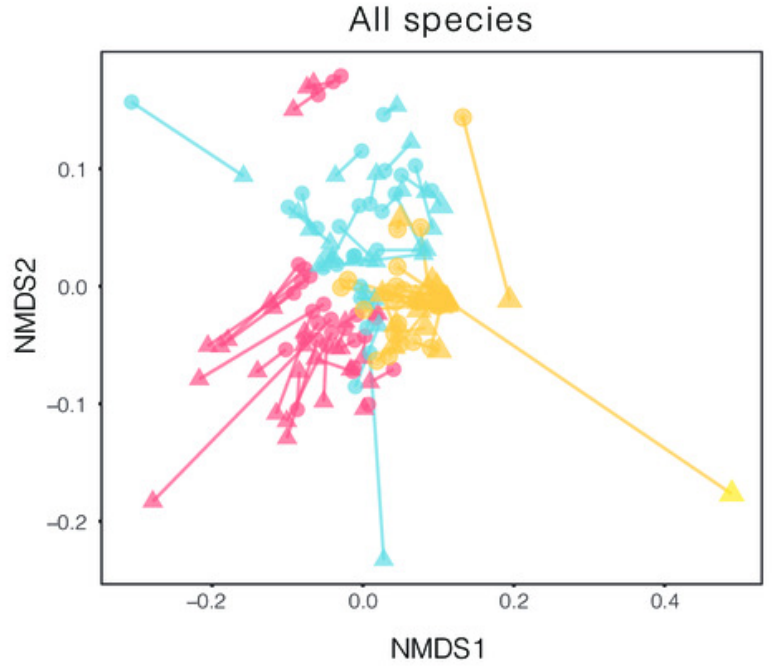

Valencia hispanica Decreasing Imesing

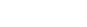

2.381 381 $\rightarrow-\infty=$
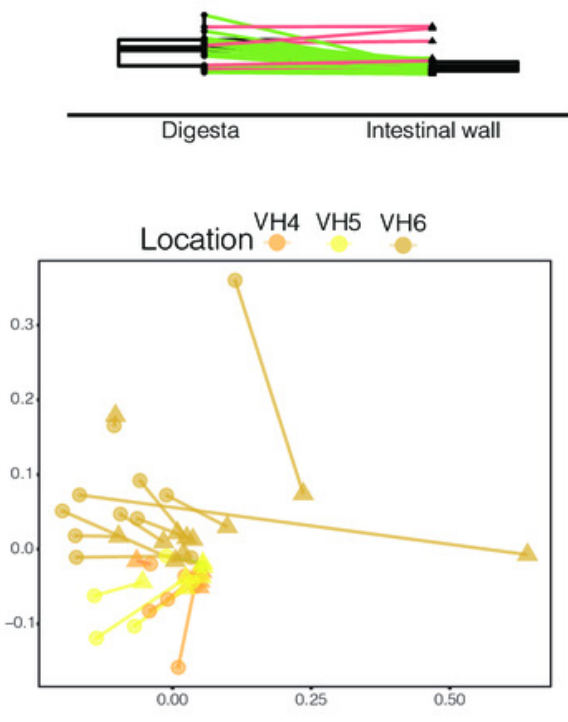

Sample type

- Digesta

$\boldsymbol{\Delta}$ Intestinal wall

Species

- Aphanius iberus

- Gambusia holbrooki

Valencia hispanica 


\section{Figure 3}

Enriched bacterial families in the log-normal submodel.

For simplicity only families that are more abundant in intestinal wall samples compared to digesta samples with strong (red) or moderate (orange) statistical support or families more abundant in digesta samples compared to intestinal wall samples with strong (blue) statistical support are shown. Families that are significantly more abundant in either intestinal wall or digesta samples in one species of fish, but not significant in another species of fish are shown as a blank space. Plot showing all families can be found in supplemental material (Fig. S5). 


\section{Enriched microbial families}
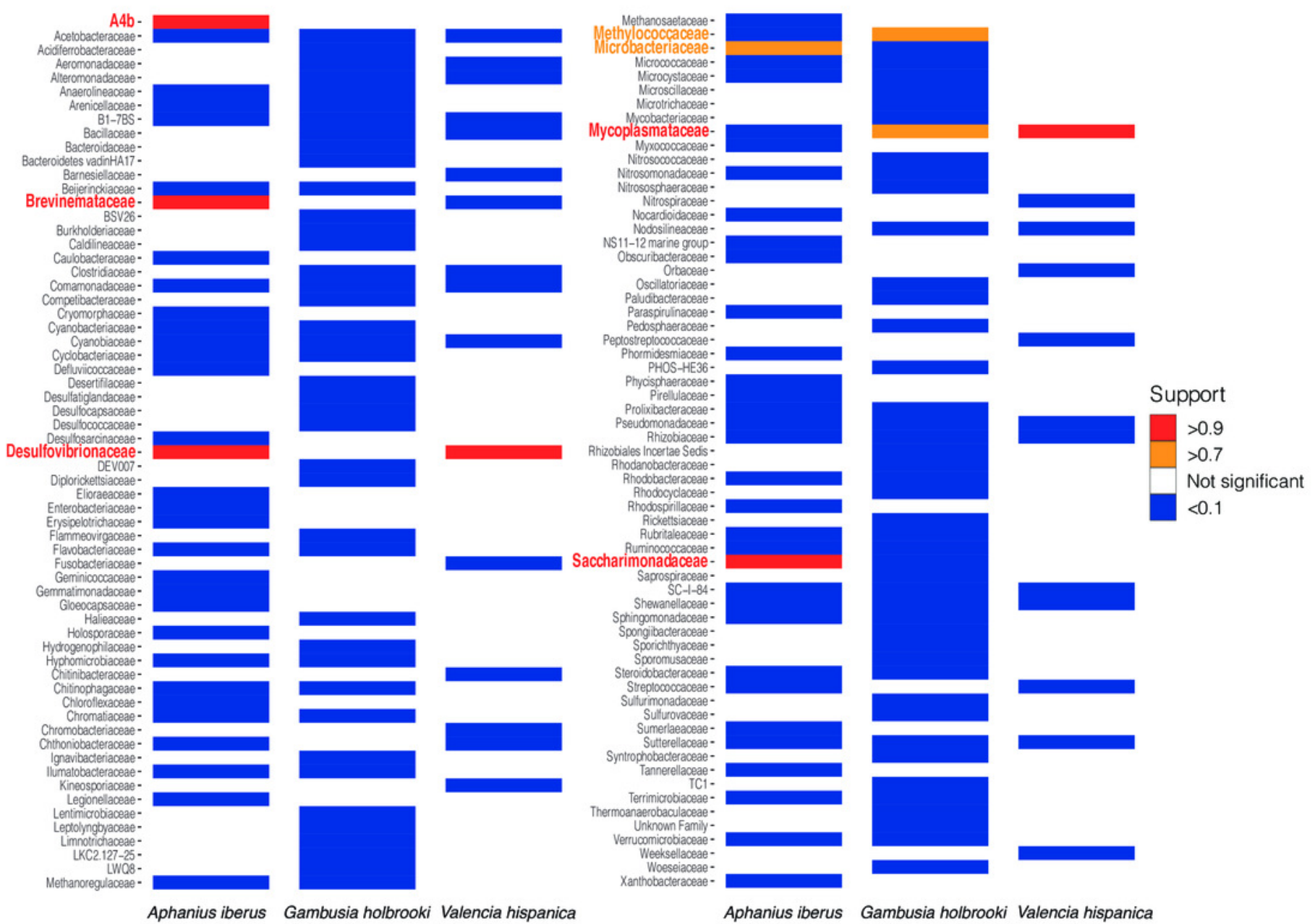

Aphanius iberus Gambusia holbrooki Valencia hispanica

Aphanius iberus Gambusia holbrooki Valencia hispanica 